\title{
Produção científica brasileira fundamentada na Teoria de Enfermagem de Orem: revisão integrativa
}

\author{
Brazilian scientific production based on Orem's Nursing Theory: integrative review \\ Producción científica brasileña basada en la Teoría de Enfermería de Orem: revisión integradora
}

\section{Maria Lúcia Raimondo', Débora Fegadoli', Marineli Joaquim Méier", Marilene Loewen Wall", Liliana Maria Labronici"I', Maria Isabel Raimondo-Ferraz ${ }^{\mathrm{IV}}$}

'Universidade Federal do Paraná, Programa de Pós-Graduação em Enfermagem (Mestranda),

Grupo de Estudos Multiprofissional em Saúde do Adulto. Curitiba-PR, Brasil.

"Universidade Federal do Paraná, Curso de Graduação em Enfermagem, Programa de Pós-Graduação em Enfermagem. Curitiba-PR, Brasil.

I' Universidade Federal do Paraná, Curso de Graduação em Enfermagem, Programa de Pós-Graduação em Enfermagem,

Grupo de Estudos Multiprofissional em Saúde do Adulto. Curitiba-PR, Brasil.

IV Universidade Federal do Paraná, Programa de Pós-Graduação em Enfermagem (Doutoranda),

Grupo de Estudos Multiprofissional em Saúde do Adulto. Curitiba-PR, Brasil.

Submissão: 14-07-2010 Aprovação: 30-07-2012

\section{RESUMO}

Revisão integrativa, realizada nas bases de dados LILACS, SciELO e BDENF, de janeiro de 2005 a maio de 2009, com o objetivo de sumarizar a produção científica brasileira fundamentada na Teoria de Enfermagem de Orem. Obtiveram-se 23 artigos, analisados pela estatística descritiva simples. Constatou-se que $100 \%$ dos estudos se voltaram para adultos. Deste total, $65,22 \%$ voltavam-se aos portadores de patologias crônicas. Em 39,15\% das pesquisas, a teoria foi utilizada na íntegra e em $34,80 \%$ um dos construtos. $91,30 \%$ das publicações objetivaram a construção e implantação da prática de cuidado estruturada e teoricamente fundamentada. Concluiu-se que a teoria vem sendo utilizada como base teórico-filosófica para fundamentar a práxis da Enfermagem em uma multiplicidade de situações, de modo a salientar o papel do enfermeiro no cuidado.

Descritores: Enfermagem; Teoria de Enfermagem; Autocuidado.

\section{ABSTRACT}

Integrative review, held in the databases LILACS, SciELO and BDENF from January 2005 to May 2009, aimed to summarize the Brazilian scientific production based on Orem's Nursing Theory. We obtained 23 articles, analyzed by simple descriptive statistics. It was found that $100 \%$ of the studies focused on adults. Of this total, $65,22 \%$ returned to the chronicle diseases. In $39,15 \%$ of the searches, the theory was used in full and in $34,80 \%$ one of the constructs. $91,30 \%$ of publications aimed to the construction and deployment of the structured and theoretically grounded practice of care. It was concluded that the theory has been used as theoretical and philosophical basis to justify the practice of nursing in a variety of situations in order to emphasize the role of the nurse in the care. Key words: Nursing; Nursing Theory; Self Care.

\section{RESUMEN}

Revisión integradora, que se celebró en las bases de datos LILACS, SciELO y BDENF, de enero 2005 a mayo 2009, con el objetivo de resumir la producción científica de enfermería basada en la Teoría de Orem. Se obtuvieron 23 artículos, analizados por estadística descriptiva simple. Se encontró que el 100\% de los estudios se volvió hacia los adultos. De este total, $65,22 \%$ volvieron a las enfermedades crónicas. En 39,15\% de las búsquedas, la teoría fue utilizada en su totalidad y en 34,80\% una de las construcciones. $91,30 \%$ de las publicaciones se destinaran a la construcción y implantación de la práctica del cuidado estructurada y fundamentada teóricamente. Se concluyó que la teoría se ha utilizado como base teórica y filosófica para justificar la práctica de la enfermería en una variedad de situaciones con el fin de destacar el papel de la enfermera en el cuidado.

Palabras Clave: Enfermería; Teoría de Enfermería; Auto cuidado. 


\section{INTRODUÇÃO}

A Enfermagem durante muitos anos teve uma orientação dirigida para enfrentar apenas as circunstâncias técnicas imediatas de modo intuitivo. Todavia, a influência de diversos fatores como os científicos, sociais, culturais, políticos e econômicos remeteram a Enfermagem a um processo de reflexão acerca do ser e do fazer profissional.

Na década de 1950, surgiram muitos questionamentos em torno do agir tecnicamente orientado, quando então os enfermeiros passaram a enfatizar a aplicação de princípios científicos nos seus procedimentos. Nessa mesma época, surge a necessidade de desenvolver um corpo de conhecimento específico que pudesse conferir identidade e autonomia à profissão. É neste contexto que se passou a elaborar os princípios que norteariam teoricamente o agir profissional ${ }^{(1)}$.

Em sua história, a Enfermagem mostrou-se dependente de outras ciências sem que houvesse um corpo de conhecimento próprio, o que fomentou o desejo nos enfermeiros de conhecer sua verdadeira natureza e construir sua identidade ${ }^{(2)}$. Destarte, em função de sua consolidação como uma ciência focada no cuidado ao ser humano, a Enfermagem necessita compreender a filosofia do cuidar e sua utilização nas ações que requerem situações terapêuticas ${ }^{(3)}$.

O advento das teorias é compreendido como uma preocupação dos profissionais de enfermagem em imprimir orientação teórica que possibilite a sistematização da prática e o desenvolvimento de atividades apoiadas em processos científicos, que lhe permita refletir e avaliar suas ações, de modo a aprimorar sua prática. Observa-se que a elaboração de teorias reflete a necessidade dos profissionais comporem um corpo de conhecimentos específicos da profissão, a fim de que possa ser reconhecida como ciência ${ }^{(4)}$.

A necessidade de conhecer e produzir seu próprio conhecimento levou a Enfermagem especialmente, a norte-americana, a investir tempo e esforços no desenvolvimento de teorias, modelos e marcos conceituais próprios da profissão, utilizados como instrumental para direcionar sua prática profissional e estabelecer as bases de seu conhecimento(4).

As teorias podem ser entendidas como formas de representação da realidade e definidas como um conjunto de proposições utilizadas para descrever, explicar e predizer parte de uma realidade. Elas consistem na organização de algum fenômeno por meio da qual se evidenciam os componentes e as características que Ihes conferem identidade ${ }^{(2)}$, e têm sido um passo fundamental em direção à compreensão da Enfermagem como práxis, como ação aprofundada pela reflexão, carregada de sentido, projetada, consciente e transformadora da natureza, do homem e da sociedade ${ }^{(5)}$.

Apesar de toda evolução da ciência, percebe-se ainda certa dificuldade por parte do enfermeiro em apoiar seu fazer nas teorias, seja no âmbito da assistência, ensino ou pesquisa. Nesse sentido, destaca-se a importância da aplicação de marcos teóricos para construção do conhecimento ${ }^{(6)}$. A visão caridosa que ainda permeia o cuidar da Enfermagem deve dar lugar a uma visão reflexiva de natureza filosófica, pautada nos princípios éticos, epistemológicos e ontológicos da profissão como ciência do cuidado(3), capaz de produzir conhecimentos que subsidiem sua prática profissional.

O termo teoria refere-se a uma generalização abstrata que apresenta uma explicação sistemática de como os fenômenos estão inter-relacionados. Seus principais objetivos são: dar significado aos resultados científicos; resumir o conhecimento existente em sistemas coerentes; estimular novas pesquisas; fornecer direção ao estudo, bem como, explicar a natureza das relações entre as variáveis. Sua utilização proporciona ao enfermeiro o conhecimento necessário para aperfeiçoar sua prática ${ }^{(7)}$.

Uma das teorias consideradas como marco teórico de referência para a prática profissional do enfermeiro é a Teoria Geral de Enfermagem proposta por Orem. Essa teórica apresentou suas concepções de Enfermagem, em 1959, quando publicou pela primeira vez seu conceito de enfermagem como provimento do autocuidado. Em 1971, publicou Nursing: Concepts of Practice, onde centralizou seu foco no indivíduo, e em 1980, publicou a segunda edição reformulada, de modo a incluir unidades multipessoais como família, grupos e comunidade ${ }^{(8)}$.

Em 1985, foi lançada a terceira edição apresentando então a Teoria Geral de Enfermagem de Orem, formada por três construtos teóricos relacionados, sendo eles: o autocuidado, as deficiências do autocuidado e os sistemas de enfermagem. Em 1991, apresentou sua quarta edição incorporando aos seus escritos maior ênfase na criança, nos grupos e na sociedade ${ }^{(8)}$.

Orem enfatiza a importância do engajamento do cliente para o autocuidado, para possibilitar que indivíduos, família e comunidade tomem iniciativas e assumam responsabilidades no desenvolvimento efetivo de seu próprio cuidado em direção à melhoria da qualidade de vida, saúde e bem-estar ${ }^{(9)}$.

Percebe-se ser inegável e amplo o avanço da Enfermagem no que diz respeito à prestação de cuidados fundamentados em princípios teóricos científicos, porém, apesar de sua importância, tem havido por parte dos enfermeiros, questionamentos quanto à aplicabilidade das teorias na prática e, frequentemente, ouvem-se referências às dificuldades de operacionalização, no âmbito de sua atuação profissional (2).

Diante desta problemática em torno da utilização das teorias de Enfermagem, torna-se relevante analisá-las, a fim de que se possa compreender sua aplicabilidade na prática da Enfermagem. Neste sentido, faz-se o seguinte questionamento: A Teoria de Enfermagem de Orem é utilizada na pesquisa brasileira? A partir disso, desenvolveu-se uma pesquisa do tipo revisão integrativa, com o objetivo de sumarizar as produções científicas fundamentadas na Teoria de Enfermagem de Orem no desenvolvimento do cuidado.

\section{MÉTODO}

Para a consecução do objetivo proposto, realizou-se uma revisão integrativa da literatura, visto que possibilita sumarizar as pesquisas já publicadas e obter conclusões a partir de um tema de interesse ${ }^{(10)}$.

As etapas que possibilitaram a operacionalização da revisão foram as seguintes: seleção dos termos utilizados, estabelecimento dos critérios para a composição da amostra, 
triagem dos artigos, leitura e coleta dos dados, análise e interpretação dos resultados ${ }^{(11)}$.

O levantamento bibliográfico foi realizado nas bases de dados LILACS, SciELO e BDENF, com três descritores controlados junto à base da BIREME (DeCS): "enfermagem", "teoria de enfermagem", "autocuidado", e dois não controlados: "Teoria do autocuidado" e "Teoria do autocuidado de Orem". A combinação dos termos foi uma estratégia de busca utilizada nas bases de dados.

Os critérios utilizados para a seleção da amostra foram: artigos publicados em periódicos nacionais, indexados nas bases de dados LILACS, SciELO e BDENF, e que abordassem a Teoria de Enfermagem de Orem. A pesquisa possui como corte temporal o período de janeiro de 2005 a maio de 2009, momento em que finalizou-se a disciplina do Programa de Pós-Graduação para a qual este estudo foi desenvolvido.

Ao final da busca, 23 artigos correspondiam ao objetivo proposto, os quais foram analisados quanto às variáveis: ano de publicação; sujeitos da pesquisa; objetivos da pesquisa; a aplicação da teoria na prática e as considerações dos autores acerca de sua utilização. Os dados de cada estudo foram extraídos através de um formulário elaborado para esta finalidade e, posteriormente, analisados por meio da estatística descritiva simples.

\section{RESULTADOS E DISCUSSÃO}

Considerando o período pesquisado, foram selecionados 39 artigos publicados. Destes, 16 foram excluídos porque se repetiam nas diferentes bases de dados, restando, portanto, 23 artigos científicos que compuseram o corpus de análise da pesquisa. Observa-se no gráfico 1 a distribuição destas publicações.

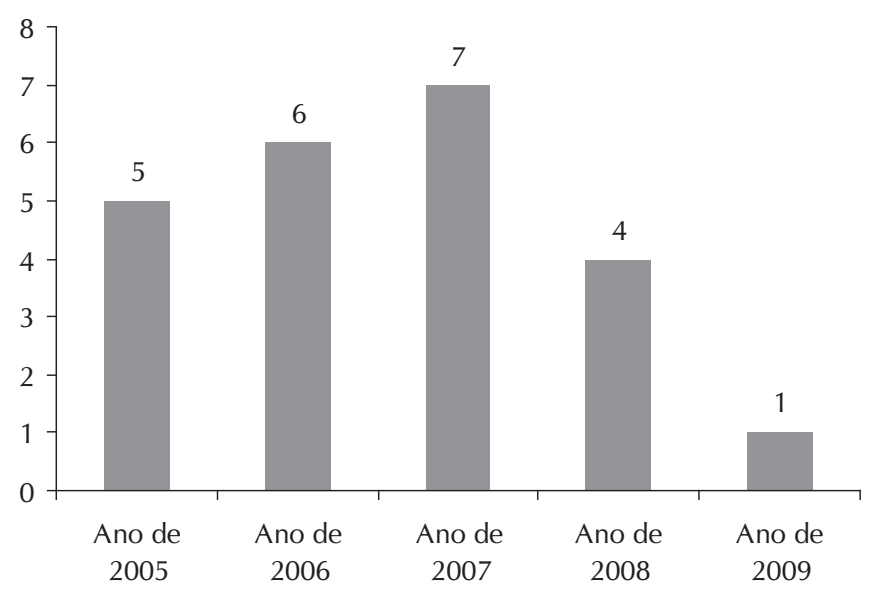

Gráfico 1 - Artigos analisados que abordavam a Teoria de Enfermagem de Orem por ano de publicação.

O ano de 2005 concentrou 21,74\% do total de artigos; 2006 com 26,09\% das produções; em 2007 publicou-se 30,43\%, sendo o ano com maior número de trabalhos; em 2008 registrou-se uma ligeira queda, com 17,39\%. O ano de 2009 concentrou apenas 4, 35\% das publicações dos últimos cinco anos, o que pode ser atribuído ao fato de que se considerou no estudo os artigos publicados apenas nos primeiros cinco meses deste ano.
$\mathrm{Na}$ atual pesquisa, constatou-se que a Teoria de Orem tem norteado o fazer da Enfermagem brasileira, considerando-se que em todos os anos pesquisados foi identificado publicações sobre a temática. O ano de 2007 concentrou o maior número de artigos, porém, chama-nos atenção que nos cinco primeiros meses do ano de 2009 somente foi encontrado um estudo utilizando esta teoria. Estes achados convergem com os encontrados em um estudo desenvolvido em 2008, onde revela uma frequência relativamente baixa de publicações que utilizam as teorias de enfermagem, que representam apenas $4,6 \%$ do total de publicações gerais de enfermagem em periódicos brasileiros. O referido estudo denota ainda que a Teoria do Autocuidado de Orem, assim como a Teoria de Enfermagem Humanística de Paterson e Zderad, e a Teoria do Cuidado Cultural de Leininger configuram os modelos conceituais mais pesquisados no

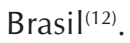

Compreende-se, desta forma, que a Enfermagem necessita incrementar a produção de conhecimentos por meio da pesquisa para maior visibilidade, reconhecimento e consolidação da profissão como ciência, tecnologia e inovação. A competência investigativa é necessária para o desenvolvimento sócio-político e tecnológico em saúde, e primordial na formação, na prática e nos centros de investigação da área ${ }^{(13)}$. Embora já existam várias teorias que podem nortear o fazer da Enfermagem, a prática do cuidar ainda é um desafio para a profissão, pois cada pessoa possui valores e princípios próprios, formação acadêmica diferenciada que acabam por influenciar o processo de cuidar ${ }^{(3)}$.

O presente estudo evidenciou que, em 100\% das publicações, o cuidado baseado na Teoria de Orem voltou-se para pessoas adultas. Em 65,22\% eram portadores de patologias crônicas ou permanentes; $13,04 \%$ pacientes/clientes com necessidades de cuidados transitórios ou temporários, e 21,74\% destinaram-se a pessoas com necessidades de cuidado-educação, conforme exposto no gráfico 2 .

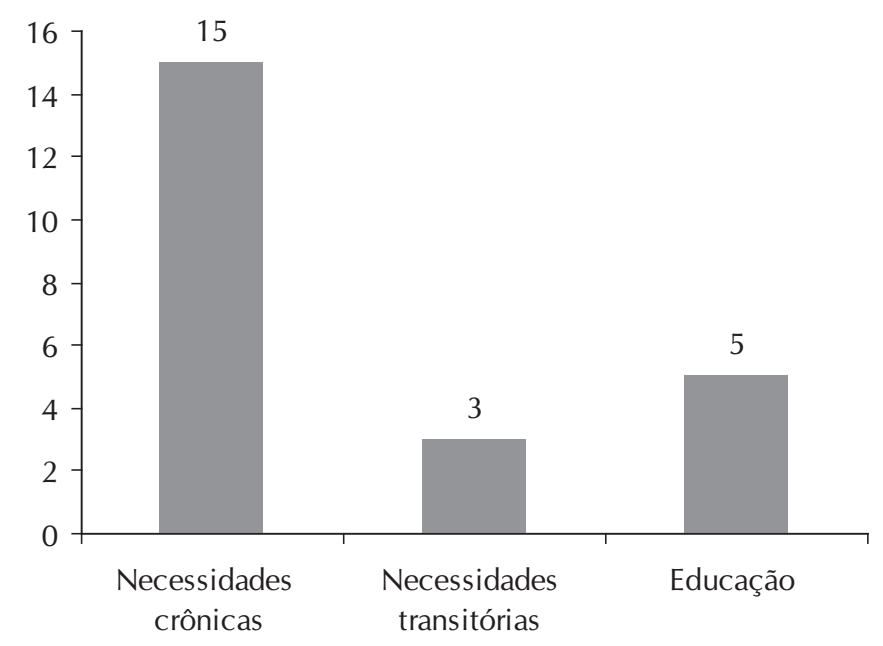

Gráfico 2 - População alvo do cuidado de Enfermagem fundamentado na Teoria de Orem identificada nos artigos analisados 
Dentre os pacientes com necessidades crônicas de cuidado, destaca-se que 13,05\% eram portadores de paraplegia em função de lesão medular; $13,05 \%$ doentes renais crônicos; 8,69\% diabéticos; 8,69\% ostomizados; 8,69\% hipertensos; 4,35\% cardiopatas; $4,35 \%$ portadores de necessidades especiais, e 4,35\% portadores de HIV. Já, em relação às necessidades transitórias, 4,35\% voltavam-se às mulheres grávidas; 4,35\% dependentes de álcool, e 4,35\% estudantes com cefaleia. As ações de educação e prevenção em saúde $(21,74 \%)$ destinavam-se a idosos saudáveis e famílias.

Os dados desta pesquisa revelam que a Teoria de Orem foi utilizada para atender uma gama de sujeitos portadores de patologias manifestas, todavia, com capacidade para participar ou até mesmo para desenvolver seu próprio cuidado. Fato, que condiz com o proposto pela teórica, vez que para ela, a condição que resulta na existência de uma necessidade de ações de enfermagem para um adulto é a ausência da habilidade para manter continuamente o autocuidado, que é terapêutico, na sustentação da vida e da saúde, na recuperação da doença ou da lesão, ou no enfrentamento dos seus efeitos ${ }^{(14)}$.

Na perspectiva de Orem, os sujeitos são agentes de autocuidado que se diferenciam dos outros seres vivos, porque têm a capacidade de refletir sobre si mesmos, bem como, de desenvolverem ou participarem de seu próprio cuidar. Sob esta ótica, os indivíduos devem ser autossuficientes e também responsáveis pelas suas próprias necessidades de cuidados, ou satisfazer as necessidades de outras pessoas na família que não são capazes de cuidarem de si próprios ${ }^{(9)}$.

Do total de estudos publicados, no que concerne aos objetivos apresentados pelos pesquisadores, 91,30\% visavam à construção e implantação de uma prática de cuidar estruturada e teoricamente fundamentada, como demonstrado no gráfico 3 .

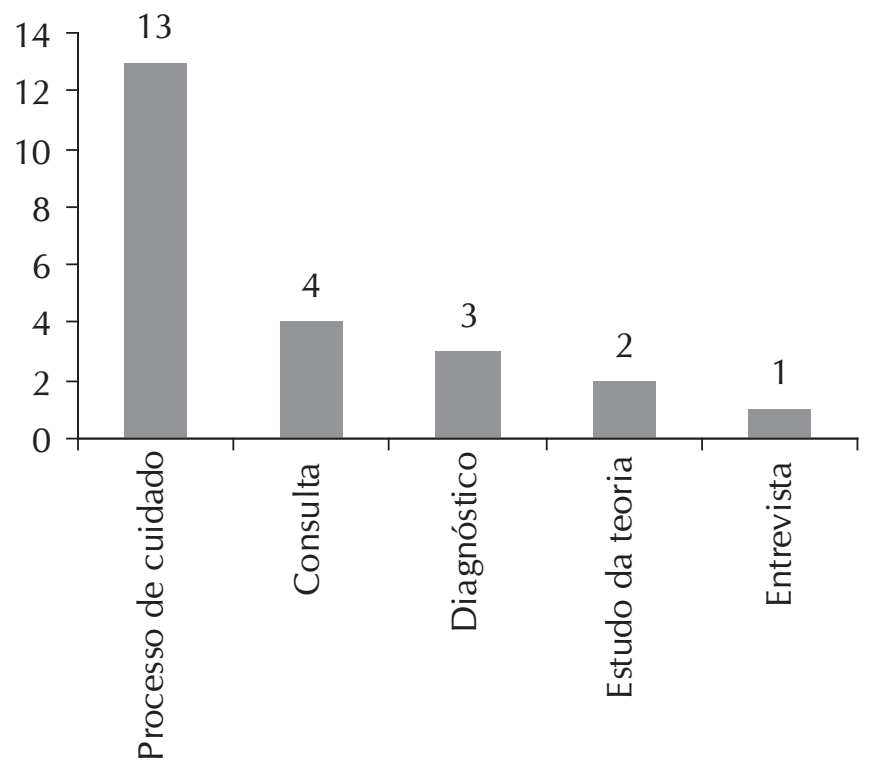

Gráfico 3 - Objetivos apresentados nas pesquisas que utilizaram a Teoria de Orem.
$\mathrm{Na}$ maioria dos estudos $(56,50 \%)$, o objetivo proposto foi a elaboração e implantação do Processo de Enfermagem a pacientes com necessidades de cuidado de enfermagem, com vistas a preparar o paciente para o autocuidado; $17,40 \%$ tiveram por objetivo a estruturação da Consulta de Enfermagem; $13,05 \%$ utilizaram partes da teoria como apoio para elaboração de Diagnósticos de Enfermagem; 8,70\% tinham a finalidade de analisar e aprofundar conhecimentos acerca da Teoria de Orem, como reflexões e revisões de literatura, contudo, sem aplicação prática; 4,35\% visaram à construção de um roteiro de entrevista a ser aplicada em momentos diversificados do processo de cuidar.

A prática diária de cuidado de enfermagem deve ser teoricamente embasada e construída na intersecção entre o campo da filosofia, da ciência e técnologia e da ética, em uma abordagem epistemológica comprometida com a sociedade ${ }^{(3)}$, com o que alcançará o almejado reconhecimento profissional.

As teorias de Enfermagem podem ser consideradas aportes epistemológicos fundamentais para a construção do saber e da prática profissional, vez que auxiliam na orientação dos modelos clínicos e possibilitam que os profissionais descrevam e expliquem aspectos da realidade assistencial, auxiliando no desenvolvimento da tríade: teoria, pesquisa e prática na área ${ }^{(12)}$.

Acredita-se que a escolha pela Teoria de Orem pelos enfermeiros se deva ao fato de que este referencial possibilita, a partir de uma ação também educativa do enfermeiro, o desenvolvimento de atitudes que facultem aos indivíduos e/ou grupos populacionais o autocuidado, bem como ao avanço das condições crônicas das doenças que exigem dos indivíduos o uso de tratamentos, tecnologias no domicílio e a reestruturação de estilos de vida, como formas de cuidado ${ }^{(12)}$.

Ao que tange à forma de aplicação da teoria em foco à prática do cuidado, esta pesquisa revela que os enfermeiros lançam não da teoria completa ou apenas partes da mesma para fundamentar o cuidado, como pode ser observado no gráfico 4 .

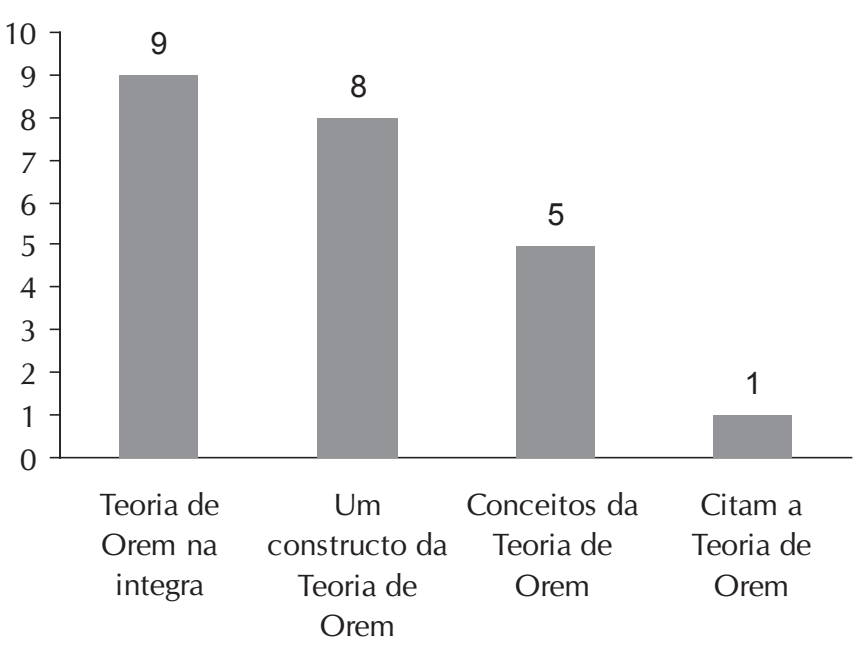

Gráfico 4 - A forma de utilização da Teoria de Orem pelos pesquisadores nos artigos analisados. 
Em relação à forma como a teoria foi utilizada nas pesquisas, constatou-se que $39,15 \%$ dos artigos analisados aplicaram a teoria na íntegra. Em 34,80\% das publicações, os autores usaram um dos construtos, prevalecendo a Teoria do Autocuidado. Em $21,75 \%$ das pesquisas apenas foram utilizados conceitos para fundamentar algumas atividades de cuidado de enfermagem, e em $4,35 \%$ a teoria foi apenas citada na pesquisa.

De maneira geral, as teorias se estruturam a partir de quatro conceitos centrais: ser humano, saúde, meio ambiente e enfermagem, o que torna relevante considerar que o conhecimento produzido precisa levar em conta o compromisso social da profissão ${ }^{(15)}$. A Teoria Geral de Enfermagem de Orem é formada por três construtos teóricos inter-relacionados: a Teoria do Autocuidado, a Teoria do Déficit de Autocuidado e a Teoria dos Sistemas. Assim, pode ser utilizada na íntegra, pondo-se em prática os três construtos concomitantes, ou em partes, optando-se pelo construto que melhor atenda as necessidades no planejamento do cuidado de enfermagem ${ }^{(9)}$.

A Teoria do Autocuidado: caracterizada pela prática das ações de cuidado executadas pelos pacientes por si mesmos para manter, promover, recuperar ou conviver com os efeitos e limitações das alterações de saúde. A Teoria do Déficit de Autocuidado: momento em que o enfermeiro atua como provedor de autocuidado, face às habilidades insuficientes do paciente para satisfazer as suas demandas terapêuticas. A Teoria dos Sistemas de Enfermagem: Neste momento, Orem classifica três sistemas de enfermagem para os requisitos de autocuidado: Sistema Totalmente Compensatório, no qual o paciente, em função de suas ações estarem limitadas, é incapaz de realizar o autocuidado; Sistema Parcialmente Compensatório, onde o enfermeiro e o paciente realizam medidas de cuidado; Sistema Apoio-educação, no qual o paciente realiza e regula suas atividades de autocuidado e o enfermeiro auxilia para que este seja um agente de autocuidado ${ }^{(9)}$.

As suposições empregadas na teoria de Orem são aceitas pela comunidade de enfermagem, uma vez que seus conceitos são aplicáveis e úteis para explicitar a importância do paciente e as relações entre este e a Enfermagem ${ }^{(16)}$.

Um ponto importante a ser levado em consideração, é que a Teoria de Orem é contemporânea com o conceito de promoção e manutenção de saúde da população, vez que prevê ações de promoção e educação em saúde. Quando empregada no cuidado, ela tem a capacidade de definir quando a Enfermagem se faz necessária e quando o paciente pode desenvolver seu próprio cuidado ${ }^{(17)}$. Nesta perspectiva, considera-se que as teorias foram elaboradas para explicitarem a complexidade e multiplicidade dos fenômenos presentes no campo da saúde e, também, para servirem como referencial teórico/metodológico/prático aos enfermeiros que se dedicam à construção de conhecimentos, ao desenvolvimento de investigações e à assistência no âmbito da profissão(15).

Esta revisão buscou levantar as considerações expressas pelos autores acerca da utilização desta teoria na prática profissional. Observou-se que $86,96 \%$ dos pesquisadores fizeram referência à importância, contribuições e as possibilidades do uso da teoria, das quais se destaca a visibilidade do Processo de Trabalho da Enfermagem, o respaldo à prática independente do enfermeiro, e a transformação do paciente em agente do cuidado.

$\mathrm{O}$ uso de teorias reflete um movimento da profissão em busca da autonomia para desenvolver o cuidado. Representa um dos elementos que compõem a linguagem específica elaborada pelos atores profissionais, objetivando consolidar a Enfermagem como ciência e arte na área da saúde ${ }^{(12)}$.

\section{CONSIDERAÇÕES FINAIS}

Esta pesquisa analisou a produção de conhecimento da Enfermagem brasileira em relação à utilização da Teoria de Enfermagem de Orem no período de 2005 a 2009. Concluiu-se que esta teoria, na íntegra ou em partes, vem sendo utilizada como base teórico-filosófica para fundamentar a práxis da Enfermagem em uma multiplicidade de situações, de modo a salientar o papel do enfermeiro no cuidado individual e coletivamente, uma vez que no período estudado foram identificadas publicações sobre a temática.

Os resultados evidenciaram que o ano de 2007 concentrou o maior número de artigos, porém, chama-nos atenção que, nos cinco primeiros meses do ano de 2009, somente foi encontrado um estudo utilizando esta teoria. Os estudos publicados voltaram-se predominantemente para a população adulta, sendo que $65,22 \%$ eram portadores de patologias crônicas ou permanentes; 13,04\% com necessidades de cuidados transitórios ou temporários, e 21,74\% destinaram-se a pessoas com necessidades de cuidado-educação. O objetivo principal dos estudos foi à construção e implantação de uma prática de cuidar estruturada e teoricamente fundamentada.

Na percepção dos pesquisadores, nos artigos analisados, a teoria proporciona visibilidade ao processo de trabalho da Enfermagem, respalda cientificamente a prática e possibilita a participação direta do paciente em seu próprio cuidado. Neste sentido, sugere-se que outros estudos possam ser desenvolvidos para avaliar as situações já testadas, com o que se poderá confirmar a aplicabilidade da mesma, vez que este é um importante caminho para que a Enfermagem utilize e sustente suas teorias.

\section{REFERÊNCIAS}

1. Martins MR, Gomes FV, Nishimura CH. Percepção dos discentes acerca das teorias de enfermagem num curso de graduação. Arq Apadec 2004;(8 Supl 1):788-93.

2. Almeida VCF, Lopes MVO, Damasceno MMC. Teoria das relações interpessoais de Peplau: análise fundamentada em Barnaum. Rev Esc Enferm USP 2005;39(2):202-10.

3. Andrade BB, Bellini EF, Santos MES dos, Waidman MAP. Ontologia e epistemologia do cuidado de enfermagem. Arq Ciênc Saúde UNIPAR 2008;12(1):77-82.

4. Aguillar OM, Mendes JAC. Viabilidade da aplicação de 
teorias de enfermagem: relato de experiência no âmbito da pesquisa. Rev Esc Enferm USP 1988;22(n. ${ }^{\circ}$ esp):47-52.

5. Leopardi MT. Teorias em enfermagem: instrumentos para a prática. Florianópolis: Papa-Livros; 1999.

6. Oliveira TC, Lopes MVO, Araujo TL. Modo fisiológico da teoria de Roy: análise reflexiva segundo Meleis, In: Anais do 57. Congresso Brasileiro de Enfermagem; 2005 nov 03-07; Goiânia, Brasil. Goiânia: Associação Brasileira de Enfermagem; 2005.

7. Santos I, Sarat CNF. Modalidades de aplicação da teoria do autocuidado de Orem em comunicações científicas de enfermagem brasileira. Rev Enferm UERJ 2008;16(3):313-18.

8. Taylor SG. Dorothea E. Orem: la teoría enfermera del deficit de autocuidado In: Tomey AM, Alligood MR. Modelos y teorías en enfermería. Madri: Elsevier Science; 2003. p. 189-211.

9. George JB, editor. Teorias de enfermagem: os fundamentos para a prática profissional. 4. ed. Porto Alegre: Artes Médicas Sul; 2000.

10. Rodrigues RM, Bagnato MHS. Pesquisa em enfermagem no Brasil: problematizando a produção de conhecimentos. Rev Bras Enferm 2003;56(6):646-50.

11. Almeida MCP, Rodrigues RAP, Furegato ARF, Scochi CGS. A pós-graduação na Escola de Enfermagem de Ribeirão
Preto - USP: evolução histórica e sua contribuição para o desenvolvimento da enfermagem. Rev Latino-am. Enferm 2002;10(3):276-87.

12. Schaurich D, Crossetti MdaGO Produção do conhecimento sobre teorias de enfermagem: análise de periódicos da área, 1998-2007. Esc Anna Nery Rev Enferm 2010;14(1):182-88.

13. Erdmann AL, Lanzoni GMM. Características dos grupos de pesquisa da enfermagem brasileira certificados pelo CNPq de 2005 a 2007. Esc Anna Nery Rev Enferm 2008; 12(2):316-22.

14. Santos ZMSA, Oliveira VLM. Consulta de enfermagem ao cliente transplantado cardíaco: impacto das ações educativas em saúde. Rev Bras Enferm 2004;57(6):654-7.

15. Thofehrn MB, Leopardi MT. Teorias de enfermagem, trabalho e conhecimento contemporâneo. Texto \& Contexto Enferm 2002;11(1):86-104.

16. Silva IJ, Oliveira MFV, Silva SED, Polaro SHI, Radünz V, Santos EKA, et al. Cuidado, autocuidado e cuidado de si: uma compreensão paradigmática para o cuidado de enfermagem. Rev Esc Enferm USP 2009;43(3):697-703.

17. Foster PC, Bennett AM. Dorothea E. Orem. In: George JB. Teorias de enfermagem: os fundamentos à prática profissional. 4. ed. Porto Alegre: Artes Médicas; 2000. p. 83-101. 